Mots. Les langages du politique

\title{
La presse devant les attentats terroristes : usages journalistiques du mot guerre (Paris, 2015)
}

The press and terrorist attacks: how journalists use the word guerre (war)

(Paris, 2015)

Los atentados terroristas y la prensa: como los periodistas usan la palabra

guerre (la guerra) (París, 2015)

\section{Marie Veniard}

\section{OpenEdition}

Journals

Édition électronique

URL : https://journals.openedition.org/mots/23077

DOI : $10.4000 /$ mots.23077

ISSN : 1960-6001

Éditeur

ENS Éditions

Édition imprimée

Date de publication : 20 mars 2018

Pagination : $91-109$

ISSN : 0243-6450

Référence électronique

Marie Veniard, «La presse devant les attentats terroristes : usages journalistiques du mot guerre (Paris, 2015) », Mots. Les langages du politique [En ligne], 116 | 2018, mis en ligne le 23 février 2020, consulté le 23 avril 2022. URL : http://journals.openedition.org/mots/23077 ; DOI : https://doi.org/ $10.4000 /$ mots. 23077 


\section{La presse devant les attentats terroristes : usages journalistiques du mot guerre (Paris, 2015)*}

L'année 2015 fut marquée, en France, par deux séries d'attentats meurtriers ${ }^{1}$, en janvier puis en novembre. Par leur ampleur, ces derniers ont plongé les Français dans la sidération (Ory, 2016). L'ampleur de l'évènement s'est traduite par un traitement médiatique très important en termes de volume et très divers en termes de supports (voir Lefébure, Sécail, 2015). Dans cette contribution, il s'agit d'éclairer les modalités d'usage du mot guerre, qui a circulé dans les discours médiatiques et politiques comme une description possible des évènements, en janvier, puis de nouveau en novembre. On peut en effet s'interroger sur l'usage de ce mot, dont le référent prototypique est l'opposition militaire entre deux États, pour renvoyer à des attentats terroristes. Nos travaux antérieurs sur l'usage de guerre pendant le conflit qui débute en 2001 en Afghanistan nous avaient permis de constater sa circulation très rapidement après les attentats du 11 septembre aux États-Unis (Veniard, 2013). Cette similitude nous a incitée à analyser le traitement médiatique des attentats de 2015 par deux quotidiens de référence (Le Monde et Le Figaro), et, parmi de nombreux angles possibles, à nous focaliser sur leur usage opposé de ce même mot selon une double perspective, sémiotique et discursive. Nous nous situons ici dans le prolongement de travaux d'analyse de discours qui soulignent l'importance de la circulation de certains vocables, ou constructions, dans la configuration du sens des évènements (voir par exemple Moirand, 2007 ; Krieg-Planque, 2003). Nous suivrons la voie initiée par Paul Siblot, celle d'une approche située de la nomination, qui «donne un point de vue anthropologique sur l'objet nommé » (Siblot, 1996, p.138).

* Je remercie les différents relecteurs anonymes de la revue, Chloé Gaboriaux et Christine Barats, pour leurs objections et suggestions.

1. Le 7 janvier 2015, une attaque terroriste contre la rédaction du journal satirique Charlie Hebdo a causé la mort de 12 personnes, dessinateurs, collaborateurs et membres des forces de l'ordre. Peu de temps après, cet évènement a été suivi d'une fusillade et de deux prises d'otages, dont une dans une épicerie casher de la ville de Vincennes. Puis une seconde série d'attentats a eu lieu. Le 13 novembre, différents lieux culturels et sportif à Paris et à Saint-Denis ont été visés. Ces attaques ont provoqué la mort de 130 personnes. 


\section{Sens social de l'évènement et nomination}

Avec Érik Neveu et Louis Quéré (Neveu, Quéré, 1996), on parlera de configuration (ou construction) du sens social par les médias, dans la mesure où, avec leurs contraintes propres (Champagne, 2000), ces derniers sont un des opérateurs centraux du récit qui est fait des évènements et du sens qui leur est donné. Ce récit comporte une large part de discours extérieurs (discours politique, scientifique, institutionnel, syndical, etc.), que le discours médiatique fait dialoguer (Moirand, 2007). Partie prenante du récit donnant sens à l'évènement (Ricœur, 1991), la nomination apparaît comme une ressource pour comprendre celui-ci (Quéré, 2006). Tel est le rôle qu'a joué la dénomination guerre dans le traitement médiatique des attentats, parmi d'autres dénominations possibles (Moirand, Reboul-Touré, 2015).

\section{Guerre et terrorisme}

Le terme guerre renvoie à un phénomène social qui a beaucoup évolué au cours du temps (Van Creveld, 1998 [1991]). Le sens du mot a changé lui aussi (Branca-Rosoff, 1999), ce qui fait dire à John Searle que l'existence d' "une guerre dépend en partie du fait que l'on pense que c'en est une » (Searle, 1998, p. 2). Le mot combine deux acceptions, l'une juridique (la guerre est déclarée par le Parlement et encadrée juridiquement), l'autre non spécialisée (un état de violence à l'échelle nationale ou internationale). En 2015, l'usage du mot se fonde sur cette seconde acception, sans que cela n'exclue totalement la première pour renvoyer à des évènements concomitants (la guerre en Syrie par exemple). L'usage du mot est rendu encore plus complexe par sa polysémie (la guerre «armée » vs la guerre contre la pauvreté), les deux acceptions cohabitant (voir plus bas le titre «Guerre des “marques" au sein du djihadisme français »).

À l'heure actuelle, les frontières entre la guerre et le terrorisme sont floues. Pour l'historien Pascal Ory (2016, chap.v), le terrorisme relève bien d'une démarche guerrière, de type guerre civile, en ce qu'il est une forme organisée de violence. La rhétorique de la « guerre » lors d'actes terroristes n'est pas nouvelle (Koren, 1996 ; Garcin-Marrou, 2001) et l'usage de guerre en France en 2015 a bénéficié d'un précédent notable. Ainsi, lors des attentats de 2001 aux États-Unis, les dirigeants américains ont très vite qualifié les faits d'actes de guerre, ce qui a placé le pays en position de légitime défense et autorisé une réponse militaire, position avalisée par le Conseil de sécurité de l'ONU (résolution 1368, 2001). Après les attentats de novembre 2015, le président de la République française a déclaré le pays « en état de guerre » et imposé l'état d'urgence. L'armée française a par ailleurs effectué des frappes militaires contre le 
groupe État islamique en Syrie, ce qui entérine la confusion référentielle et l'indistinction des acceptions du mot (sens militaire $v s$ non militaire).

\section{Contextualisation discursive}

En 2015, dans le contexte d'attentats terroristes, la désignation guerre entre en circulation dans l'espace médiatique chargée d'une mémoire collective (Moirand, 2014), c'est-à-dire une mémoire constituée des faits et des discours tenus dans l'espace public et les médias après le 11 septembre 2001 par les hommes et femmes politiques, ainsi que par les experts. Cette mémoire, que les journalistes ont en tête et qu'ils supposent aux citoyens, légitime l'usage de ce mot. Elle est présente explicitement dans la construction du sens social des attentats de 2015 en France. Ainsi, Le Monde titre «Le 11 septembre français» dans l'édition du 9 janvier. L'expression guerre contre le terrorisme, utilisée par le gouvernement américain après les attentats de 2001 mais abandonnée par la suite (interview de Donald Rumsfeld, Tribune Media Service, 12/12/2006), a été remise en circulation en 2015 par les journalistes français. En 2001 pourtant, le président français, parmi d'autres responsables politiques, était réticent à l'égard de l'usage de ce mot :

(1) Vaste débat sémantique : faut-il parler de guerre à propos des attentats terroristes contre les États-Unis? À Washington, Jacques Chirac est interrogé sur ce point par la presse. Il finit par suggérer le mot : «conflit». À vrai dire, quelle différence entre une guerre et un conflit? De toute façon, la controverse est ridicule. Une action de force qui fait près de 6000 morts et détruit tout un quartier d'une grande ville, qu'est-ce d'autre qu'un acte de guerre? (Le Figaro, 21/09/2001)

Ces réticences peuvent avoir des explications multiples. On peut penser par exemple que le dialogisme du mot guerre en France, distinct de celui du mot war en anglais américain, aurait été moins favorable à l'usage du mot, ou que l'intensité de l'émotion ressentie était plus forte aux États-Unis qu'en France. Quoi qu'il en soit, c'est ce même mot qui a ressurgi en 2015, même si son inadéquation potentielle reste présente à l'esprit des locuteurs, qui tentent de la contourner en ayant recours au «parler vrai » (voir plus bas).

En France, comme aux États-Unis, le discours des dirigeants politiques ${ }^{2}$ a été très important dans la constitution du récit des évènements. Le compte rendu médiatique des évènements est en effet étroitement dépendant des allocutions officielles. Le 13 janvier, dans un discours à l'Assemblée nationale, le Premier ministre emploie le terme : «La France est en guerre contre le

2. L'analyse de l'usage de guerre par les dirigeants politiques français en 2015 excède largement les limites de cet article. Différents facteurs pourraient être envisagés, tels que la construction d'éthos de «chef de guerre ", les rapports de force dans le champ politique, un appel à la peur de la part du gouvernement afin de faire accepter des mesures d'état d'urgence limitant les libertés des citoyens, etc. 
terrorisme, le djihadisme et l'islamisme radical ", apportant ainsi tout le poids de sa fonction à la circulation du mot. En novembre, après une intervention le lendemain des attaques (14/11), le président s'est exprimé le 16 novembre devant le Congrès, qui réunit les deux chambres, l'Assemblée nationale et le Sénat. À ces deux occasions, il a catégorisé les évènements comme des «actes de guerre », validant l'usage de la nomination.

L'usage du mot dans le discours politique lui garantit une bonne diffusion dans le discours médiatique en raison des voix extérieures (hétérogénéité énonciative) qui traversent ce dernier (dépêches, citations, tribunes, etc.). Les commentateurs politiques, en effet, s’ils ne sont pas habilités à produire le discours politique, sont habilités à le « reproduire, [l']amplifier et [le] qualifier» (Le Bart, 1998, p. 108).

Toutefois, même si le discours politique offre un soutien important à la circulation du mot, il n'apparaît pas comme sa seule source énonciative, la désignation circulant dans la presse avant le discours de Manuel Valls du 13/01/2015. En janvier, des témoins évoquent une «scène de guerre» (Le Monde, 09/01, p. 8), tandis qu'en novembre, les médecins et les personnels soignants investis dans les secours parlent de "blessures de guerre », les policiers d' "armes de guerre » (voir par exemple Le Monde du 15-16/11 et Le Figaro du 16/11), ce qui contribue à la diffusion du signifiant.

Les évènements ont en effet suscité, chez de nombreux citoyens, des émotions telles que la peur ou la sidération. Or, si l'on suit l'analyse de Jean-François Tétu (2004), on trouve la trace de ces émotions dans des titres tels que «La terreur à Paris» (Le Monde, 15-16/11) ou "La guerre en plein Paris» (Le Figaro, 14-15/11). Pour J.-F. Tétu, l'émotion suscitée par certains évènements se marque par le dispositif, notamment le direct à la télévision, ou bien par certains procédés discursifs, l'évènementialisation par exemple, «c'est-à-dire l'exhibition d'une fracture dans l'état du monde» (Tétu, 2004, p.15). Tétu donne comme exemple le recours à l'expression guerre par certains titres français lors du 11 septembre («La nouvelle guerre» dans Le Figaro, ou «État de guerre», dans Le Point). Parler d'émotion pour ce type de titres suppose de se placer au niveau de ce que Raphaël Micheli (2014), s’appuyant sur la tradition rhétorique, appelle des émotions «étayées », c'est-à-dire des émotions que l'interlocuteur infère à partir du récit que fait le locuteur d'une situation qui lui est socioculturellement associée (Micheli, 2014, p. 29). La guerre, en particulier si elle survient dans le pays du locuteur, est susceptible de générer des émotions qui sont, conventionnellement, la peur et/ou le courage. Titrer sur la guerre revient à faire appel à ces émotions, dont l'intensité est à l'échelle de l'évènement et de son « évènementialisation ».

L'émergence de guerre dans le contexte des attaques de janvier 2015 nous semble dépendre de ces phénomènes de divers ordres, l'évolution des pratiques de la guerre, un précédent et les discours tenus à cette occasion, le tout combiné avec un évènement d'une gravité rare. Les attentats de novembre, en 
réactivant les mêmes ressorts - à ceci près que les discours tenus en janvier viennent s'adjoindre à ceux de 2001 -, confirment ce choix nominatif, comme nous le verrons dans ce qui suit.

\section{La couverture médiatique des attentats}

Nous avons choisi de travailler sur la version imprimée des journaux, qui offre la possibilité d'avoir accès à la fois à la hiérarchisation, au dispositif sémiotique complexe mis en œuvre et au discours produit, ce qui permet une description sémiotique et discursive plus complète du sens de l'évènement que ne l'autoriserait un corpus de journaux en ligne. On sait par ailleurs que les sites des journaux affichent la plupart des articles de la version imprimée et qu'ils ont été très consultés pendant cette période3.

Le corpus a été constitué à la suite d'une première phase exploratoire sur différents quotidiens nationaux et régionaux, tels que Le Figaro, Le Parisien, Le Monde, Libération et l'hebdomadaire L'Express. Un examen des unes et des premiers éditoriaux publiés après les attentats a permis d'observer des différences importantes entre Le Monde et Le Figaro.

\begin{tabular}{|c|c|c|}
\hline Journal & Attentats de janvier 2015 & Attentats de novembre 2015 \\
\hline Le Figaro & $\begin{array}{l}\text { (08/01) La liberté assassinée } \\
\text { [pied de page : l'éditorial, inti- } \\
\text { tulé «C'est la guerre»] }\end{array}$ & $(14-15 / 11)$ La guerre en plein Paris \\
\hline Le Monde & (09/01) Le 11 septembre français & $\begin{array}{l}\text { (15-16/11) [surtitre] } \\
\text { Vendredi } 13 \text { novembre, } 21 \mathrm{~h} 20 \\
\text { [titre] La terreur à Paris } \\
\text { [pied de page : reportage, } \\
\text { «C'est la guerre»] }\end{array}$ \\
\hline
\end{tabular}

Tableau 1. Titres de la première une sur l'évènement

Le Figaro utilise le mot guerre dans un des titres de la une en janvier et, en cela, il se distingue aussi bien du Monde que du Parisien ("Ils ne tueront pas la liberté ») ou de L'Express («Nous sommes la France»). En novembre, à l'inverse, Le Figaro, Le Parisien ("Cette fois, c'est la guerre») et L'Express ("Comment gagner la guerre »), par exemple, ont recours au mot dans le titre de une, tandis que Le Monde fait sa manchette sur les émotions ("La terreur»). En raison de ces choix divergents, nous nous concentrerons maintenant sur $L e$ Monde et Le Figaro, tous deux des quotidiens de référence, les plus vendus au

3. Les chiffres de vente et de consultation des pages des journaux en ligne sont disponibles sur http://www.acpm.fr/. 
plan national. Le premier est un journal qui revendique la défense des valeurs humanistes et une certaine indépendance par rapport aux partis politiques (Eveno, 2001) : il affiche une volonté de rester neutre par le choix de refléter dans ses colonnes les différents points de vue. Malgré cela, on le considère généralement comme un journal de centre gauche. Le Figaro, quant à lui, est un quotidien de la droite conservatrice 4 (Blandin, 2007).

Le corpus se compose des numéros parus entre le 8 et le 14 janvier 2015 (soit 6 pour chacun des journaux) et entre le 14 et le 19 novembre 2015 (5 pour Le Figaro, 4 pour Le Monde). Dans chaque cas, le corpus inclut les éditions rendant compte des discours tenus par le gouvernement après les attentats. Le corpus a été constitué à partir des versions imprimées originales des journaux, complétées par la base de données Europresse, qui offre un accès à la version imprimée pour certains titres.

Le mot guerre apparaît dans les titres de une mais aussi dans les éditoriaux des deux quotidiens. En janvier, seul Le Figaro en fait usage dans son éditorial en référence aux attentats. Ce journal le met très nettement en valeur dans le titre de l'éditorial, de façon d'autant plus visible que, comme dans Le Monde, ce dernier commence en page une, ce qui arrive parfois dans cette publication. Le Monde parle quant à lui d'acte de guerre, ce qui est une façon de mettre à distance la dénomination guerre, en minimisant les faits.

\begin{tabular}{l|l}
\hline \multicolumn{1}{c|}{ Journal } & \multicolumn{1}{c}{ Attentats de janvier 2015} \\
\hline $\begin{array}{l}\text { Le Figaro } \\
\text { (08/01) }\end{array}$ & $\begin{array}{l}\text { titre : La guerre } \\
\text { C'est une guerre, une vraie guerre, menée non par des soldats mais par des } \\
\text { assassins de l'ombre, des tueurs méthodiques et organisés, dont la tranquille } \\
\text { sauvagerie glace le sang. Elle a tué, hier, en plein Paris. } \\
\text { Cette guerre, longtemps nous n'avons pas voulu la voir. Elle se déroulait si loin } \\
\text { de nous, n'est-ce pas?, aux confins de la Syrie, de l'Irak, du Nigeria ou de la } \\
\text { Libye... Par scrupule - sans doute par peur aussi -, nous n'osions pas même } \\
\text { dire son nom. [...] Depuis hier, les euphémismes ne sont plus de mise : c'est } \\
\text { une guerre, une vraie guerre, qui nous a été déclarée : la guerre du fanatisme } \\
\text { islamiste contre l'Occident, l'Europe et les valeurs de la démocratie. [...] } \\
\text { [début de §] Face à la guerre, le premier devoir est de s'unir. [...] } \\
\text { [fin de §] Quand la guerre est là, il faut la gagner. }\end{array}$ \\
\hline $\begin{array}{l}\text { Le Monde } \\
\text { (09/01) }\end{array}$ & $\begin{array}{l}\text { Libres, debout, ensemble } \\
\text { [...] C'est enfin la France qui est visée, car elle est en première ligne, seule ou } \\
\text { avec ses alliés, dans la guerre engagée contre le djihadisme international. } \\
\text { [dernier §] Oui, «nous sommes tous Charlie». Au-delà de la traque engagée } \\
\text { par la police pour les retrouver, c'est la meilleure réponse que nous puissions } \\
\text { adresser aux auteurs de cet acte de guerre contre la France et les Français. } \\
\text { Nous le devons aux victimes, nos amis. }\end{array}$ \\
\hline
\end{tabular}

Tableau 2. Extraits des éditoriaux publiés le lendemain de l'attaque à la rédaction de Charlie Hebdo (janvier 2015)

4. Une enquête IFOP-Marianne sur le vote au premier tour des municipales en 2014 en fonction des «habitudes médias» révèle que $51 \%$ des lecteurs du Monde ont voté pour une liste de gauche, alors qu'à $60 \%$, les lecteurs du Figaro ont voté pour une liste de la droite ou du centre (enquête disponible sur: http://www.ifop.fr/media/poll/2588-1-study_file.pdf). 


\begin{tabular}{|c|c|}
\hline Journal & Attentats de novembre 2015 \\
\hline $\begin{array}{l}\text { Le Figaro } \\
(14-15 / 11)\end{array}$ & $\begin{array}{l}\text { Gagner la guerre } \\
\text { C'était une guerre, une vraie guerre. Nous le savions, mais au fond nous } \\
\text { préférions ne pas le voir. } \\
\text { [...] Après les sommets d'abomination barbare atteints dans la nuit de } \\
\text { vendredi à samedi, ces illusions ne sont plus de mise. C'est une guerre, une } \\
\text { vraie guerre, qui nous est déclarée : la guerre du fanatisme islamiste contre } \\
\text { la France, l'Europe, l'Occident et contre toutes les valeurs d'une civilisation } \\
\text { qui a inventé la démocratie. Cette guerre, maintenant chacun l'a compris, ne } \\
\text { fait que commencer. } \\
\text { [...] François Hollande, dans son intervention de samedi, s'est enfin décidé } \\
\text { à nommer le mal. Sans finasser, il parle - c'est nouveau - de «guerre», } \\
\text { de « "Daech» et de "djihad». Mais Manuel Valls aussi, après Charlie, avait } \\
\text { su trouver les mots. Et la suite a montré que, face aux barbares, les mots } \\
\text { n'étaient pas suffisants... } \\
\text { [...] On se souvient de Clemenceau : «En politique intérieure, je fais la guerre. } \\
\text { En politique extérieure, je fais la guerre. Je fais toujours la guerre.» Pour } \\
\text { gagner la guerre, il faut la mener. [fin du texte] } \\
\text { [Encadré au milieu de l'article] Cette guerre, maintenant chacun l'a compris, } \\
\text { ne fait que commencer. }\end{array}$ \\
\hline $\begin{array}{l}\text { Le Monde } \\
(15-16 / 11)\end{array}$ & $\begin{array}{l}\text { L'effroi et le sang-froid } \\
\text { La France est en guerre. En guerre contre un terrorisme totalitaire, aveugle, } \\
\text { terriblement meurtrier. } \\
\text { [...] Cela s'imposait, pour faire face à cette guerre que les « djihadistes» } \\
\text { veulent nous imposer. [...] } \\
\text { À la demande du gouvernement de Bagdad, Paris participe, avec une } \\
\text { cinquantaine d'autres pays, à la guerre (essentiellement aérienne) menée } \\
\text { contre l'«État islamique», qui s'est emparé d'une bonne partie du territoire } \\
\text { de l'Irak. [...] } \\
\text { Ce combat que mène Paris contre le djihadisme expose la France. [...] Il faut } \\
\text { être aveugle ou sourd pour ne pas lire et entendre le discours de l'État isla- } \\
\text { mique, d'Al-Qaïda et d'autres mouvements islamistes : ce sont des appels à } \\
\text { porter la «guerre sainte» en Europe, à tuer les «infidèles», les «juifs», les } \\
\text { "croisés». [...] } \\
\text { [dernière phrase] Rester nous-mêmes est l'une des conditions du succès } \\
\text { dans la guerre qu'il faut mener contre ce fanatisme. }\end{array}$ \\
\hline
\end{tabular}

Tableau 3. Extraits des éditoriaux publiés après les attaques de novembre 2015

L'emploi du mot par Le Figaro est stable d'un évènement sur l'autre, alors que Le Monde passe d'acte de guerre en janvier à La France est en guerre en novembre, ce qui nous invite à approfondir la comparaison entre les deux publications.

\title{
Approche sémiotique : la mise en valeur de la nomination
}

\author{
L'analyse du corpus a montré que le mot guerre n'apparaît pas simplement dans \\ les articles, mais aussi, de manière récurrente, dans des positions «visibles », \\ que l'on suppose "attractives», comme la titraille ou certains endroits spéci- \\ fiques du texte. Avant d'en venir au traitement des évènements, nous décrirons \\ au fil de cette section les différentes positions dans lesquelles on rencontre le \\ mot, en les rapportant au processus d'évènementialisation.
}




\section{Titres et évènementialisation}

La mise en valeur la plus visible s'effectue par les titres, en particulier le titre de une ou un titre présent sur la page de une. Dans les grandes rédactions, les titres, et plus généralement la titraille (surtitre, sous-titre, chapeau), sont écrits par le secrétaire de rédaction, qui s'assure ainsi de la cohérence de l'ensemble des titres sur une même page. Dans le cas le plus classique, le titre reprend un énoncé de l'article. Dans certains cas, plus intéressants quant à la mise en valeur du mot guerre, le titre constitue une reformulation plus percutante (pour reprendre l'expression d'Yves Agnès, 2002, p.147) d'un extrait de l'article5. Dans l'exemple qui suit, deux citations d'une même personne sont compilées de façon à former un titre attractif.

(2) Pour le FN, la guerre est ouverte contre l'islam radical (Le Monde, 09/01, p. 9)

La citation est sous forme de modalisation en discours second introduit par une locution du type «selon X», forme qui induit une mise à distance (AuthierRevuz, 1997). Ce titre est constitué de la synthèse de différents propos de la présidente du Front national. On peut lire d'un côté : «Selon la présidente du Front national, il y a une guerre ouverte entre le terrorisme et les démocraties»; de l'autre : "Pour elle, cela ne fait aucun doute : cette attaque a été commise "au nom de l'islamisme radical [...]" ». Le titre proposé par Le Monde radicalise, sans le déformer véritablement, le propos de Marine Le Pen. La «guerre entre le terrorisme et les démocraties » est remplacée par une désignation plus attendue et plus caractéristique de la responsable politique. Cette refonte s'inscrit dans les pratiques professionnelles des journalistes, pour lesquels le respect des propos cités n'est qu'un des paramètres qui guident l'utilisation d'une citation. Alice Krieg-Planque (2000, p. 83) relève d'autres impératifs, notamment celui de « dire ce que la personne aurait voulu dire » (mais qu'elle n'aurait pas su dire).

Parfois, plus rarement, le mot apparaît en titre mais ne figure pas dans l'article.

(3) Guerre des «marques» au sein du djihadisme français (Le Figaro, 14/01, p.19, Champs Libres)

(4) La France vote la poursuite de la guerre contre Daech en Irak (Le Figaro, 14/01, p.11)

Dans le premier exemple, l'article relate des différends entre les organisations islamistes. Le mot guerre a clairement ici un sens métaphorique, mais le signifiant, prégnant dans l'espace médiatique, se diffuse pour des référents d'une nature non militaire. Dans le second cas, le titre reformule la première

5. Maurice Mouillaud et J.-F. Tétu (1989, partie III, chap. II) recensent les modifications que peut subir un titre par rapport au contenu de l'article. 
phrase de l'article : «Un climat d'unanimité inédit a présidé mardi au vote des députés et sénateurs sur la prolongation des frappes aériennes françaises en Irak contre l'État islamique (EI), alias Daech» (nous soulignons en italique les fragments similaires). Chacun des segments soulignés est reformulé de façon plus générale dans le titre.

Ces cas sont plus rares, mais ils mettent en évidence la position privilégiée du terme dans un titre, ce qui souligne la dimension d'attracteur du mot et tend à confirmer le procédé d'évènementialisation mis en œuvre par les journaux.

\section{Le péritexte : un autre espace de mise en visibilité}

La mise en valeur apparaît à travers d'autres lieux péritextuels (surtitre, soustitre, intertitre), qui offrent moins de visibilité que le titre, mais plus que le texte de l'article lui-même. La mise en valeur passe par une police de caractères de taille supérieure à celle utilisée dans les articles et/ou par un décalage spatial : citations ou extraits en encadré au milieu d'un article (voir annexe 1), ou série d'informations brèves ou de citations en bandeau ou en colonne.

(5) [Titre] Le pape dénonce «des formes déviantes de la religion» [encadré] "Une vraie guerre mondiale qui se déroule par morceaux»

[Source du titre et de l'encadré dans l'article] Citant également le Pakistan où "plus de cent enfants ont été tués avec une férocité inouïe» il y a un mois, le chef de l'Église catholique a établi ce diagnostic : c'est «comme une vraie guerre mondiale qui se déroule par morceaux» où des "formes déviantes de la religion» jouent un rôle certain. (Le Figaro, 13/01, p. 7)

(6) Le Tweet. La guerre a été déclarée à la France, à ses institutions, à la République, par des barbares qui nient l'idée même de civilisation et les valeurs universelles de l'humanisme. @NicolasSarkozy (Le Figaro, 10-11/01, p. 2, en colonne)

Le titre et l'encadré, se détachant du texte, fonctionnent de concert. Dans l'exemple (5), une citation du pape a été distribuée entre le titre et l'encadré. Si le titre n'est pas surprenant, puisqu'on attend du pape qu'il se positionne sur la question de la religion, l'encadré, plus martial, est moins attendu. On peut penser que l'effet recherché, la surprise, résulte ici de la juxtaposition d'énoncés relevant de registres différents (religieux vs martial). On peut relever également que, dans le changement de lieu sémiotique, une partie de la citation a été perdue : l'adverbe comme, qui modalisait la référence de guerre. Par conséquent, dans l'encadré, la référence est directe, et le propos plus assertif (voir plus bas l'analyse de l'adjectif vrai). L'exemple (6) se caractérise également par son caractère martial (déclarer la guerre), équilibré par un appel aux valeurs (la République, l'humanisme).

Ces dispositifs participent de la recherche de "vi-lisibilité» (Adam, Lugrin, 2000) dans la maquette, ce qui s’inscrit dans la tendance actuelle à 
l'éclatement des articles en différents modules. Ils permettent de prévenir un « rejet de l'écrit» (Mouriquand, 2015 [1997], p. 23), en rendant le contenu accessible plus rapidement. La présence dans ces modules du mot guerre, plus précisément, d'énoncés comportant ce mot, est un indice de l'attractivité prêtée au mot et de son rôle dans le processus d'évènementialisation à l'œuvre.

\section{Mise en valeur intratextuelle}

Le mot peut être mis en valeur par des positions privilégiées à l'intérieur du texte lui-même, à savoir le début, ou accroche, qui doit donner «envie» de lire (Mouriquand, 2015 [1997], p. 116), et la fin, la chute du texte. L'éditorial du Figaro (08/01, voir tableau 2) se caractérise par une présence du mot dans ces deux positions de mise en valeur discursive.

Nous proposons en annexe 2 des extraits d'une double-page Opinions (Le Monde, 17/11/2015) qui illustrent de manière exemplaire ces procédés. Le mot y apparaît, notamment en début de texte, dans deux constructions syntaxiques récurrentes qui soulignent l'acte de catégorisation : la thématisation (c'est la guerre) et la construction attributive ( $X$ est une guerre/un acte de guerre). Le caractère exceptionnel du référent est renforcé par une référence fréquemment déictique, qui signale la dimension partagée de l'évènement (on dit la guerre, celle qui est en train de se dérouler et que tout le monde connaît dans ce contexte).

Le genre des articles n'est pas anodin : le mot apparaît préférentiellement dans des articles d'opinion (voir l'annexe 2), tribunes rédigées par des extérieurs ou chroniques de journalistes de la rédaction, ainsi que dans les articles consacrés à la politique française (discours du chef de l’État, action du gouvernement, réactions de la classe politique). Il est quasi absent des articles consacrés à la traque des criminels ou aux explications contextuelles (parcours des terroristes, radicalisation des jeunes, etc.).

Nous avons montré, au fil de cette section, que le mot guerre apparaît dans des positions de mise en valeur. Nous proposons d'expliquer cette présence par le fait que les journalistes considèrent ce mot comme participant du processus d'évènementialisation, c'est-à-dire de sur-marquage de l'évènement, ce qui est manifeste dans les cas où le mot n'apparaît que dans le titre, ainsi que dans les énoncés catégorisants appuyant une prise de position dans les tribunes.

\section{Comparaison du traitement des attentats dans Le Monde et Le Figaro}

D'un point de vue quantitatif, on note une augmentation de la fréquence du mot entre les attentats de janvier et ceux de novembre, dans les deux jour- 
naux. On ne doit pas surinterpréter cette augmentation : les référents sont différents (assassinat ciblé vs massacre de masse), tout en se succédant, et les journaux ont peut-être adopté des stratégies différentes lors des deux évènements (autocensure, ou au contraire, affichage du mot). Nous insisterons plutôt sur l'usage du mot, qui ouvre sur des interprétations plus riches sémantiquement. La comparaison' 6 s'est effectuée à partir de l'observation des deux modes de fonctionnement du mot : la place dans les titres, en tant que mode d'évènementialisation, et la prise en charge énonciative du mot par le journal.

\section{Guerre dans les titres}

Dans Le Monde, entre le 9 et le 13 janvier 2015, le mot se caractérise par une quasi-absence dans les titres (2 occurrences seulement). La mise en valeur est assurée par le péritexte hors titre (6 occurrences), parfois de manière massive, comme le 10/01: sur les 5 occurrences de guerre que comporte cette édition, 3 figurent dans le péritexte. En janvier, la désignation n’apparaît pas centrale dans le récit du sens de l'évènement produit par les journalistes. La situation est bien différente en novembre puisque le mot figure plusieurs fois en une sous la forme :

- d'un titre de reportage au discours direct : “ “C'est la guerre” ( $15-16 / 11$, article qui se poursuit en pages intérieures);

- d'un titre de pages d'opinion, «Un pays face à la guerre» (17/11, même type d'article);

- d'un appel de titre : «Ces Européens qui ne croient pas à la guerre» (19/11). Nous voyons dans le premier titre un argument assez net en faveur de la thèse du caractère attractif du mot guerre et de l'évènementialisation qu'il permet. En effet, dans l'édition du 15-16/11, on peut lire ce titre en bas de la une :

Reportage / "“C'est la guerre” / Par Florence Aubenas / [premier paragraphe de l'article]

Cet article est disposé sur une colonne seulement et le titre au discours direct sans énonciateur explicite peut être interprété comme «ce que tout un chacun se dit à ce moment-là en France ». Mais la suite de l'article en page intérieure porte un titre différent, moins frappant : " "Cette nuit, la ville aussi, ils l'ont tuée" ". Les deux titres sont fondés sur des citations d'anonymes, tirées de ce papier d'ambiance sur l'état d'esprit des badauds à Paris après les attentats. Ici, les contraintes de maquette et les contraintes d'attractivité de la une se sont rencontrées pour mettre en avant le mot guerre en une.

6. Nous avons procédé à un comptage manuel indicatif des occurrences en excluant les lexies avec guerre (guerre sainte), les syntagmes avec un nom tête autre que guerre quand ils ne renvoient pas aux attentats (budget de guerre, effort de guerre), les occurrences de guerre avec des référents différents (Deuxième Guerre mondiale, ou la guerre contre le groupe État islamique menée en Syrie depuis août). 
D’une manière générale, on compte, en novembre, dans Le Monde, un nombre plus important de titres comportant le mot guerre (9 titres, dont 5 le $17 / 11$ ), tels que «État de guerre : jusqu'où peut aller François Hollande? ». Le nombre de mises en valeur dans le péritexte (hors titre) augmente légèrement ( 6 en janvier, 10 en novembre, mais avec un doublement du nombre d'occurrences du mot).

Dans Le Figaro, au contraire, la désignation est nettement adoptée, comme l'ont montré les titres de une et les éditoriaux cités plus haut. Dès janvier, le mot est en usage et assumé par le journal : on compte 9 occurrences dans le premier éditorial. Sur une période de 6 éditions (incluant le compte rendu du discours de M. Valls), le nombre de titres incluant guerre (4 occurrences) est légèrement supérieur à ce qui est observé dans Le Monde. C'est très variable selon les jours. La mise en valeur dans le péritexte (hors titre), en revanche, est similaire à celle produite par Le Monde.

En novembre, le nombre de titres faisant figurer guerre a plus que doublé. Le mot est affiché en manchette le lendemain des attentats : «La guerre en plein Paris». Il figure dans 10 titres en 4 jours (14-19/11), dont, par exemple : «Faire la guerre sans l'aimer» (16/11) ou "Comment faire la guerre à l'État islamique » (16/11). La mise en valeur est accentuée dans les titres, mais n'augmente pas significativement dans le péritexte (hors titres).

Pour conclure sur ce point, on peut dire que la mise en valeur du mot est plus marquée en novembre qu'en janvier, et ce davantage dans Le Figaro que dans Le Monde. Nous chercherons à approfondir ces résultats par l'étude de l'usage du mot au sein des articles.

\section{L'usage de guerre}

Dans cette section, nous distinguons la prise en charge du mot par le journal, à travers son usage assumé et non modalisé, et la mise à distance, quelle qu'en soit sa valeur (argument d'autorité, refus d'assumer, etc.), par différentes formes d'hétérogénéité énonciative ou de réflexivité (discours rapporté, modalisation autonymique), auxquelles nous ajoutons, sans les confondre, les occurrences qui figurent dans des tribunes signées par des extérieurs. Nous en déduisons le degré d'acceptation du mot par le journal (voir Veniard, 2013, chapitre v, pour des précisions méthodologiques).

L'analyse de l'usage de la désignation confirme et précise les éléments mis au jour par l'analyse des titres. D’une manière générale, dans les deux journaux, l'usage de la désignation est dépendant des voix extérieures : le mot figure souvent dans un contexte d'hétérogénéité énonciative, spécialement dans un intertexte politique et dans des tribunes. Cette présence est très marquée dans Le Monde et s'accompagne de mise à distance. Ainsi, le 09/01, en dehors de l'éditorial (et hors citations), la seule occurrence assumée par la 
voix du journal est fortement modalisée : «La France serait en guerre» (p. 8). Cet énoncé est caractéristique d'une réticence devant l'usage de ce mot, réticence qui peut prendre sa source dans l'absence d'adéquation référentielle. En novembre, en revanche, si la désignation figure également très souvent dans un contexte d'hétérogénéité énonciative, certaines occurrences sont assumées un peu plus explicitement, particulièrement dans les titres et les éditoriaux. Le ton de ces derniers devient plus martial, comme en témoignent les titres : «Libres, debout, ensemble» en janvier et «L'effroi et le sang-froid» en novembre. On peut donc parler, entre janvier et novembre, d'une acceptation progressive avec une importante mise en scène de voix extérieures au journal.

Dans Le Figaro, si l'on observe une mise à distance à travers la présence du mot dans des contextes d'hétérogénéité énonciative, les cas d'usage assumé sont beaucoup plus nombreux que dans Le Monde et s'affirment avec le temps, entre janvier et novembre.

Pour résumer, dans Le Monde, la désignation est fortement mise à distance en janvier, pour être adoptée en novembre, même si un contexte d'hétérogénéité énonciative demeure présent, alors que Le Figaro l'assume pleinement plus souvent, dès les attentats de janvier, et cela même si, dans cette publication également, la désignation figure régulièrement dans des propos rapportés.

\section{Dialogisme et « parler vrai »}

Autre signe que la nomination des évènements comme guerre ne va pas de soi : de nombreux actes explicites de catégorisation peuvent être relevés. Cette contradiction n'est qu'apparente au vu de l'hétérogénéité du discours de presse, tant au niveau du genre des articles qu'au niveau des auteurs et voix qui s'y expriment. L'acte de nommer est réalisé de manière explicite et guerre est choisi, par rapport à d'autres dénominations possibles pour poser la catégorie, comme si les dénominations en usage, attentats, attaque, n'étaient pas adéquates.

(7) Le serveur lui dit [à une jeune fille] : « Maintenant, rallume ton portable, ma belle et tu vas la perdre, ton innocence. Dans ce monde-ci, c'est la guerre». [...] Un autre : «ça ressemble à ça quand c'est vraiment la guerre». (Le Monde, 15-16/11, p.10)

(8) Tahar Ben Jelloun, écrivain :

[Début du texte] L'attaque de Charlie Hebdo est un fait de guerre. (Le Monde, 09/01, p.15, Débats)

(9) Edgar Morin, sociologue :

[Début du texte] Ce ne sont plus des attentats. Avec une action meurtrière massive menée en six lieux simultanés, la stratégie, donc la guerre est entrée dans Paris. (Le Monde, 17/11, p. 32-33, Opinions) 
La catégorisation porte soit sur un référent vague, auquel les locuteurs renvoient par des déictiques (ce monde-ci, c'est, ça), soit sur un référent déjà catégorisé (attentats, attaque) qui est renommé en tant que guerre. Dans ces extraits, on relève que ce nouveau nom, guerre, peut faire l'objet d'une insistance (vraiment). De manière récurrente, l'acte de nommer est réalisé avec un commentaire métadiscursif portant sur l'évaluation de la qualité de la désignation proposée. En suivant P. Siblot (2001) et Roselyne Koren (2013), nous parlerons de "parler vrai » pour désigner ces actes explicites de nomination par lesquels le locuteur revendique le droit ou le courage d' «appeler un chat un chat » et s'affranchit explicitement, ici, de la règle de l'objectivité journalistique. La nomination est actualisée sans modalisation visant à l'atténuer, au contraire. Les éditoriaux cités dans le tableau 2 contiennent déjà des formes de «parler vrai », nous ne les reprenons pas ici.

Le parler vrai peut s'exprimer à travers l'affirmation du lien référentiel : c'est le cas de vraie guerre, syntagme dans lequel l'adjectif redouble la relation de dénomination en assurant la conformité du référent au signifié (voir aussi : «c'est vraiment la guerre »). Il peut également s'exprimer à travers un commentaire métadiscursif explicite critiquant les «euphémismes» et les finasseries, voire la mauvaise volonté de certains à accepter la réalité (voir : «il faut être aveugle ou sourd pour [...] », Le Monde, 15-16/11; voir aussi les éditoriaux dans les tableaux 2 et 3). Tous ces énoncés contribuent à affirmer la légitimité de la nomination guerre et soulignent le courage nécessaire pour poser cet acte de nomination (voir : "nous savions mais nous ne voulions pas voir ", Le Figaro, 08/01, extrait de l'éditorial cité dans le tableau 2). Dans les seuls éditoriaux, ce parler vrai est présent dès janvier dans Le Figaro, tandis qu'il n'apparaît qu'en novembre dans Le Monde, ce qui renforce l'opposition entre les deux quotidiens.

À travers ces actes de nomination, les locuteurs «courageux» s'opposent à d'autres qui le seraient moins et qui défendraient d'autres positions. L'insistance sur la catégorisation, ainsi que le fait que certains énoncés comportent des marqueurs dont le fonctionnement est nettement dialogique (oui, vrai, bien), laisse penser qu'un discours contraire auquel ces locuteurs s'opposent est possible, voire en circulation dans l'espace public et médiatique.

(10) Appel de titre vers un article du Figarovox : «Oui, nous sommes en guerre : en finir avec les fantasmologues» (Le Figaro, 17/11, p. 19)

(11) Il n'empêche, c'est bien une guerre qui a lieu. Le dernier avatar du djihad islamiste, l'autoproclamé État islamique, le revendique. [...] Mais il ne faut pas se tromper sur la nature du combat à mener. Il ne relève pas de la guerre classique. (Le Monde, 17/11, éditorial)

Dans certains cas (10), évoquant une polémique récurrente sur les causes de la délinquance, le locuteur renvoie explicitement au groupe des «fantasmo- 
logues», à savoir ceux qui défendraient «la culture de l'excuse qui pose systématiquement l'idée que la violence exprime un mal-être, une injustice, une oppression » (on peut d'ailleurs penser que Le Monde est visé). À ceux-là s'opposent des locuteurs qui revendiquent l'usage du mot guerre pour un référent qui n'est pas nouveau et qui aurait dû depuis longtemps être nommé ainsi. Ce lieu commun discursif est caractéristique du Figaro. On en trouve la trace dans l'éditorial du 08/01 (tableau 2) ainsi que dans les extraits suivants:

(12) Pour gagner, contre les soldats du califat, cette guerre que l'on se décide enfin à nommer, il faudra surtout du courage, de la force et de la détermination. (Le Figaro, 12/01, éditorial)

(13) Chantal Delsol, professeur de philosophie politique :

[Début] II aura fallu que la France soit frappée au cœur pour forcer les Français à reconnaître les réalités de l'ennemi et de la guerre, interdites de paroles et de conscience depuis si longtemps. (Le Figaro, 13/01, p. 20, Champs Libres)

La spécificité du Figaro, comparé au Monde, tient également au fait qu'est agitée une menace, celle de la guerre civile, qui naît de la nature «interne » du terrorisme :

(14) Chronique de Nicolas Baverez:

Les objectifs poursuivis par El sont clairs. Tout d'abord, terroriser les Français et encourager le développement d'un climat de guerre civile dans notre pays. (Le Figaro, 16/11, p. 23)

(15) Interview de Gilles Kepel, enseignant à Sciences Po et spécialiste de l'islam :

Une étape a indéniablement été franchie même si, globalement, l'État islamique cherche toujours à terroriser la population, à la fracturer et à créer les conditions d'une guerre civile qui aboutirait, selon ses vœux, à la destruction de l'Europe, de l’Occident, et à la victoire djihadiste. (Le Figaro, 16/11, p. 24)

Nous ne donnons ici que quelques extraits, mais ils sont nombreux dans Le Figaro, alors que Le Monde ne fait que relayer cette thèse à travers des citations - éventuellement critiques - ou quelques articles d'opinion, notamment un article de Gilles Kepel, qui est un des promoteurs de cette thèse dans les médias. L'éventualité d'une guerre civile donne lieu à une polémique décrite par Ruth Amossy (Amossy, 2015). L'auteure identifie des groupes opposés sur le fait de savoir si la société française serait (ou non) divisée et assaillie par un «ennemi de l'intérieur » islamiste radical. Certains essayistes ou commentateurs médiatiques partisans d'une droite dure considèrent même que la France est actuellement en situation de guerre civile - l'éditorialiste au Figaro Ivan Rioufol a ainsi publié en mars 2016 un ouvrage intitulé La guerre civile.

Le Monde et Le Figaro présentent un certain nombre de similarités dans l'utilisation du mot guerre dans ces deux évènements, notamment une augmentation de sa présence dans les titres et son emploi dans des textes relevant du 
commentaire (éditorial, chronique et tribune). L'usage est toutefois plus affirmé dans Le Figaro que dans Le Monde. Cette dernière publication adopte en effet un ton plus martial et accentue l'usage du mot lors des attentats de novembre, mais c'est principalement à travers des séquences d’hétérogénéité énonciative. Le sens social donné au mot est également différent : si la guerre naît en janvier pour Le Monde, elle existait auparavant pour Le Figaro et menace même désormais de devenir une guerre civile.

L'analyse de la circulation du mot guerre dans deux quotidiens lors des attentats de 2015 fait ainsi apparaître que son usage se développe au croisement de logiques de presse et du processus d'élaboration du sens des évènements. Ces déterminations hétérogènes sont bien identifiées par l'analyse située de la nomination. D'un côté, l'analyse sémiotique a permis de mettre en évidence que ce mot participe du processus d'évènementialisation en fonctionnant comme un attracteur, en témoigne son utilisation dans la mise en page, dans les titres notamment. Une analyse quantitative serait une suite possible, qui permettrait de mesurer l'extension de ce phénomène. D'un autre côté, on a pu montrer que le mot guerre a toute sa place dans une activité de commentaire et qu'il participe au travail de configuration du sens social de l'évènement engagé par les acteurs. Nous avons de plus mis en évidence une différence d'usage assez nette entre Le Monde et Le Figaro : ce dernier a plus vite employé et assumé la nomination guerre, tandis que le premier ne l'a adoptée qu'en novembre, notamment par le prisme des citations. On peut voir là, c'est du moins l'hypothèse que nous faisons, une trace de l'éthique du Monde, qui revendique rigueur et distance dans le traitement de l'actualité7. La mise à distance du mot (par le conditionnel, par la construction acte de guerre en janvier et plus encore par sa présence dans de nombreuses citations) serait à mettre au compte d'un jugement d'inadéquation référentielle. Devant la répétition et la gravité des faits de novembre, le journal fait malgré tout usage du mot avec modération. Il est tout aussi délicat de proposer une explication unique à cette utilisation du mot par Le Figaro. Sur un plan discursif et local, l'existence dans ce journal, en parallèle, d'une polémique concernant l'éventualité d'une guerre civile en France constitue un terrain favorable à l'implantation du mot. D'un point de vue plus large, l'adoption du mot par Le Figaro pourrait relever de l'identité du journal, puisque nous avions repéré une tendance similaire en 2001 (Veniard, 2013), piste qui pourra faire l'objet de travaux ultérieurs.

7. Voir le hors-série qui compile les « règles de rédaction » du journal, Le style du Monde (2002, p. 48). 


\section{Références}

ADAM Jean-Michel, LUGRIN Gilles, 2000, «L'Hyperstructure : un mode privilégié de présentation des évènements scientifiques? " , Les carnets du Cediscor, nº 6, p. 133-149.

AGNÈs Yves, 2002, Manuel de journalisme. Écrire pour le journal, Paris, La Découverte.

Amossy Ruth, 2015, "Les fonctions de la polémique dans l'espace public contemporain », Conférence au colloque «Discours d'Europe, discours sur l'Europe», 17-18 décembre 2015.

AUtHIER-ReVuz Jacqueline, 1997, «Modalisation autonymique et discours autre : quelques remarques», Modèles linguistiques, $\mathrm{n}^{0} 35, \mathrm{p} .33-51$.

BLANDIN Claire, 2007, Le Figaro. Deux siècles d'histoire, Paris, Armand Colin.

BOURDON Jérôme, 2009, Le récit impossible. Le conflit israélo-palestinien et les médias, Bruxelles, De Boeck.

BRANCA-Rosoff Sonia, 1999, "Dictionnaires et sens social du mot guerre aux XVII et XVIII e siècles », dans L'armée au xvIII e siècle (1715-1789), G. Goubier-Robert éd., Aixen-Provence, Publications de l'université d'Aix-en-Provence, p.67-79.

Champagne Patrick, 2000, "L'évènement comme enjeu », Réseaux, nº 100, p. 403-426. EVEno Patrick, 2001, Le journal Le Monde. Une histoire d'indépendance, Paris, Odile Jacob. Garcin-MARrou Isabelle, 2001, Terrorisme, médias et démocratie, Lyon, Presses universitaires de Lyon.

KOREN Roselyne, 2013, "La critique du discours des "anciens" médias "mise au Net" : un nouveau type d'argumentation politique? ", Argumentation et analyse du discours, nº 10, http://journals.openedition.org/aad/1463 (consulté le 08/12/2017).

- 1996, Les enjeux éthiques de l'écriture de presse et la mise en mots du terrorisme, Paris, L'Harmattan.

KRIEg-PLANQUE Alice, 2003, «Purification ethnique ». Une formule et son histoire, Paris, CNRS Éditions.

- 2000, «Analyser le discours de presse. Mises au point sur le "discours de presse" comme objet de recherche», Communication, n०20 (1), p.75-97.

LE BART Christian, 1998, Le discours politique, Paris, PUF.

LefÉBURE Pierre, SÉCAILClaire éd., 2015, Le défi Charlie. Les médias à l'épreuve des attentats, Paris, Lemieux éditeur.

MıCHELI Raphaël, 2014, Les émotions dans les discours. Modèle d'analyse, perspectives empiriques, Louvain-la-Neuve, De Boeck - Duculot.

MoIRAND Sophie, 2014, "L'évènement “saisi” par la langue et la communication », Cahiers de praxématique, nº63, http://journals.openedition.org/praxematique/2362 (consulté le 08/12/2017).

- 2007, Les discours de la presse quotidienne. Observer, analyser, comprendre, Paris, PUF.

MoIRAn D Sophie, Reboul-TourÉ Sandrine, 2015, « Nommer les événements à l'épreuve des mots et de la construction du discours ", Langue française, nº 188, p. 105-120.

MouIlLAud Maurice, TÉTu Jean-François, 1989, Le journal quotidien, Lyon, Presses universitaires de Lyon.

MOURIQUAND Jean, 2015 [1997], L'écriture journalistique, Paris, PUF, 5 édition.

Neveu Érik, QuÉRÉ Louis éd., 1996, Le temps de l'évènement I [numéro thématique], Réseaux, $\mathrm{n}^{0} 75, \mathrm{p} .7-133$.

OrY Pascal, 2016, Ce que dit Charlie. Treize leçons d'histoire, Paris, Gallimard. 
QuÉRÉ Louis, 2006, «Entre fait et sens, la dualité de l'évènement », Réseaux, nº 139, p. $183-218$.

RICÆUR Paul, 1991, «Évènement et sens ", Raisons pratiques, n² 2, p. 41-56.

SEARLE John R., 1998, La construction de la réalité sociale, Paris, Gallimard.

SIB LOT Paul, 2001, «De la dénomination à la nomination. Les dynamiques de la signi-

fiance nominale et le propre du nom », Cahiers de praxématique, n³6, p.189-214.

- 1996, "Un nom, cela explique bien des choses», Modèles linguistiques, n 17, p.125139.

TÉTu Jean-François, 2004, "L'émotion dans les médias : dispositifs, formes et figures », Mots. Les langages du politique, $\mathrm{n}^{0} 75$, p. 9-19.

VAN CREVELD Martin, 1998 [1991], Les transformations de la guerre, Paris, Éditions du Rocher.

VENIARD Marie, 2013, La nomination des évènements dans la presse. Essai de sémantique discursive, Besançon, Presses universitaires de Franche-Comté.

\section{Annexes}

\section{Annexe 1}

\section{En Europe, l’extrême droite est à l'affût}

De Berlin à Londres, plusieurs partis xénophobes dénoncent les dangers de l'islam

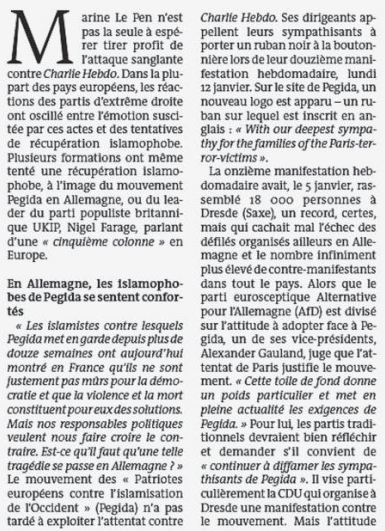

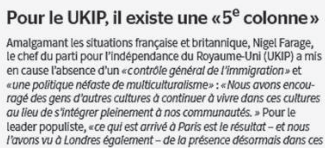

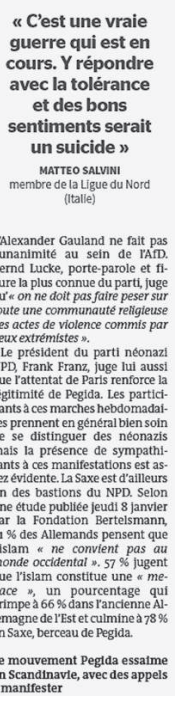

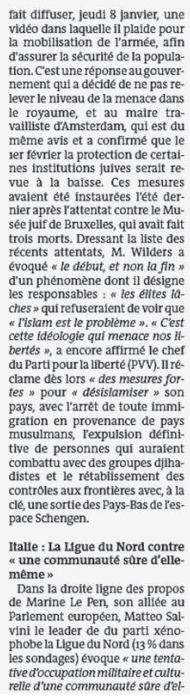

VERBATIM

66 Vous voyez ce qui arivive en Europe. Tout change radicaHollande a envoyé l'armée dans les rues. Aujourd'hui, à Paris, un massacre s'est produit avec au moins douze morts. Etici, certage limmigration illegale et: promettent la naturalisation. Le Syriza est sur une autre planete, I veut accorder massivement la a couverture sociale a tous les immigres illegaux. ?

Antonis Samaras, premier

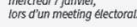

également le pape François à cesser de e dialoguer avec I'Istam " expliquant que cela " $n$ e rend pas Glovanni Toti, coordinateur de Forza Italta, le parti de Silvio Berlusconi, demande, quant a lui, a changernotre facon de deffendre nos valeurs: ie multiculturalisme
et la génerosité nont pas produit de grands resultats en Europe *. Ambigu comme à son habitude, eppe Gillo, rondateur du Mouprimatur sur son blog a un post ecrit par un universitaire dans lequel son auteur lalsse percer ses soupcons d'une manipulation et cas de lassassinat de John Ken-
nedy, rattentat de Plazza Fontana
a Milan (en 1969), lassassinat

Le Monde, 10/01/2015, p. 15, Service international ${ }^{8}$

8. Nous remercions Le Monde de nous avoir permis de reproduire cet article à titre gracieux. 


\section{Annexe 2}

Pour illustrer l'ensemble des positions attractives attribuées au mot, nous proposons un extrait du Monde daté du 17/11/2015.

Le Monde, 17/11, p. 32-33, Opinions :

Titre global : La France face à la guerre

1. Titre : Contre la terreur à Paris, il faut gagner la paix au Moyen-Orient (Edgar Morin, sociologue)

Début: Ce ne sont plus des attentats. Avec une action meurtrière massive menée en six lieux simultanés, la stratégie, donc la guerre est entrée dans Paris.

2. Titre : Prenons garde à un nouveau Munich (Jean-Noël Jeanneney, historien)

Début : Soit. C'est la guerre. Spécifique certes, dispersée, multiforme, insaisissable. La guerre pourtant, à coup sûr, avec son cortège d'horreurs.

3. Titre : Le terrorisme est la guerre de notre temps (Pascal Ory, historien)

Début : Prononcé encore avec réticence au mois de janvier, le mot «guerre » impose sa présence au mois de novembre.

Encart : La guerre est fondamentalement un acte de société : elle est la rupture organisée d'un ordre, lui-même par définition organisé. 